\title{
Economic evaluation of sacubitril/valsartan versus enalapril in the treatment of heart failure with reduced ejection fraction
}

\section{Haiqiang SANG ( $\sim$ shqXNE2@163.com )}

Zhengzhou University First Affiliated Hospital https://orcid.org/0000-0002-9467-0392

\section{Yaohui JIANG}

First Affiliated Hospital of Zhengzhou University

\section{Zhe WANG}

Zhengzhou University First Affiliated Hospiatl

\section{Rujie ZHENG}

Zhengzhou University First Affiliated Hospital

\section{Research article}

Keywords: sacubitril/valsartan, enalapril, heart failure, cost-effectiveness analysis

Posted Date: April 15th, 2020

DOI: https://doi.org/10.21203/rs.3.rs-21177/v1

License: (c) (i) This work is licensed under a Creative Commons Attribution 4.0 International License. Read Full License 


\section{Abstract}

Background: In 2020, sacubitril/valsartan(formerly LCZ696) will implement the new negotiated price of medical insurance in China, and the cost of treatment will be significantly reduced. The aim of study is to evaluate the economy of sacubitril/valsartan(SAC/VAL) compared with an angiotensin converting enzyme inhibitor (ACEI) (enalapril) in the treatment of heart failure with reduced ejection fraction (HFrEF) in China.

Method: A Markov model was developed to project clinical and economic outcomes of SAC/VAL versus enalapril for 64-year-old patients with HFrEF over 10 years from the Chinese medical and health system perspective. A cost-utility analysis was performed mostly based on data from the PARADIGM trial. Other transition probability, costs, and utilities were obtained from published literature and public databases. The primary outcome were total and incremental costs and quality-adjusted life years (QALYs) and the incremental cost-effectiveness ratio (ICER) for SAC/VAL relative to enalapril. The model was verified the uncertainty using the sensitivity analysis furtherly.

Results: Compared with enalapril, SAC/VAL cost more than enalapril ( $¥ 96532$ vs. $¥ 34560$ ) and was more cost-effective (4.6 QALYs vs. 4.3 QALYs), resulting in an incremental cost-effectiveness ratio of $¥ 185720$ per QALY gained for patients with HFrEF at a WTP threshold of $¥ 212676$ per QALY. Sensitivity analysis demonstrated the robustness of the model, identifying the death on the SAC/VAL group as a significant drivers of the cost-effectiveness. At the national negotiation price ( $¥ 9.95$ per $100 \mathrm{mg}$ ), probability of SAC/VAL being cost-effective was about $53 \%$ at a WTP threshold of $¥ 212676$ per QALY.

Conclusion: SAC/VAL was associated with clinical benefit and may be cost-effective compared with an ACEI (the current standard of care) in patients with HFrEF.

\section{Background}

Heart failure is a serious manifestation and a terminal stage of various heart diseases, which has become an increasingly serious global public health problem ${ }^{[1]}$. In 2003, the incidence rate of heart failure was $0.9 \%$ in China, when 4 million people had heart failure ${ }^{[2]}$. With the aging of population in China, the incidence rate of chronic diseases such as coronary heart disease and hypertension has been increasing. The improvement of medical level has prolonged the survival time of heart disease patients, which makes the prevalence and mortality of heart failure increased significantly. In recent years, data from all over the country shows that the prevalence of heart failure in China has increased to about $2 \%$, and about 8-10 million patients are suffering from the disease ${ }^{[3]}$. Heart failure accounts for about $40 \%$ of deaths and $20 \%$ of hospitalizations because of cardiovascular diseases, and is the second highest cause of death in China ${ }^{[4]}$. The total direct and indirect costs related to heart failure annually were estimated about $\$ 5.4$ billion in 2012 for China ${ }^{[5]}$, which will add a huge economic burden to Chinese medical security system. Although great progress has been made in the treatment of heart failure in the 
past 30 years, the case fatality rate is still as high as $50 \%$ in 5 years, and more than $50 \%$ of discharged patients need to be hospitalized again in the next 6 months ${ }^{[6,7]}$.

As a common type of heart failure, heart failure with reduced ejection fraction (HFrEF) is treated based on the use of ACEl or angiotensin receptor antagonists (ARB) combined with $\beta$ - adrenergic receptor blocker and aldosterone receptor blocker to inhibit the renin-angiotensin-aldosterone system, which can reduce the mortality of HFrEF patients. SAC/VAL is a novel anti-heart failure drug which is a part of angiotensin receptor-neprilysin enzyme inhibitor (ARNI). PARADIGM-HF (the Prospective Comparison of ARNI (angiotensin receptor-neprilysin inhibitor) with ACEI (angiotensin-converting enzyme inhibitor) to Determine Impact on Global Mortality and Morbidity in Heart Failure Trial $)^{[8]}$ trial shows that compared with enalapril, SAC/VAL reduces the risk of cardiovascular death and hospitalization of heart failure in HFrEF patients by $20 \%$. Therefore, the European heart failure guidelines in $2016^{[9]}$, the U.S. heart failure guidelines in $2017^{[10]}$ and the Chinese heart failure guidelines in $2018^{[11]}$ all recommend SAC/VAL for HFrEF. If it can tolerate ACEI / ARB, it is recommended to replace ACEI / ARB with ARNI to further reduce the risk of hospitalization and death of heart failure.

However, compared with ACEl, which costs less than $¥ 2$ per day, the application of ARNI which costs nearly $¥ 20$ per day, will significantly increase the cost of treatment. How to balance the increase of clinical benefit with the increase of treatment cost? Pharmacoeconomic evaluation is helpful to solve this problem. The economic evaluation of ARNI in the treatment of HFrEF patients has been carried out in several European countries, the United States and Singapore ${ }^{[12-18]}$,However, the medical system and economic status of these countries are different from that of China. In 2020, ARNI will implement new national negotiation prices in China, and the cost of treatment will be significantly reduced, so it is the first time in China to evaluated the economy of the treatment of HFrEF patients with SAC/VAL after the price reduction, in order to help clinicians and policy makers to judge the economic value of this new treatment.

\section{Method}

\section{Population}

Baseline characteristics, relevant data, inclusion criteria and exclusion criteria of the simulated population are all from the clinical trial of PARADIGM-HF ${ }^{[8]}$ : In this study, 8399 heart failure patients with NYHA II-IV and LVEF $\leq 35 \%$ were enrolled. The average age was 63.8 years. The median follow-up was 27 months. The average dosage of SAC/VAL was $375 \pm 71 \mathrm{mg}$, and the average dosage of enalapril was $18.9 \pm 3.4$ mg. For the unreported baseline data in the PARADIGM-HF study, they are from other published literature.

\section{Model Design}

According to the natural course of HFrEF and the published economic evaluation literature ${ }^{[12-18]}$, this study set the HFrEF patients who receive the treatment of SAC/VAL or enalapril as three states: stable 
heart failure, hospitalization for acute aggravation of heart failure and death, in which the death state was absorption state. The states were transferred according to the arrow shown in the figure (see Figure 1). The model was established by Excel 2016 software. the simulation time limit was 10 years, and the cycle was set as 1 month (30 days).

In the real world, the process of disease development, diagnosis and treatment is more complex, so some assumptions are needed in the model simulation to make the model reasonable and simplified. According to the development of HFrEF and the process of diagnosis and treatment, the following assumption were made: (1) all patients started in the state of stable heart failure before entering the longterm Markov model; (2) the effect of treatment of heart failure of SAC/VAL and enalapril was assumed not to change at any time; (3) the probability of each event during 10 years was assumed to be unchanged.

\section{Input Parameters}

\section{Transition probabilities}

If there is a cohort study of HFrEF population intervention in China, the results of the Chinese population study are preferred. All-cause mortality in 30 days after enalapril treatment of HF patients comes from the

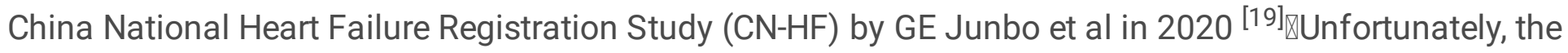
30-day hospitalization rate about HFrEF patients in China are not publicly available, Instead, data were used from PARADIGM-HF. Because the underlying data from the Kaplan-Meier curves were not presented in the publication, the necessary parameters were derived using the software tool Get Data Graph Digitizer (Braunschweig, Germany) referred to other literature ${ }^{[15,20]}$, the linear function was simulated by Microsoft Excel 2016 and Kaplan Meier curve was drawn to calculate the all-cause mortality and hospitalization rate in 30 days. The 30-day in-hospital death data and 30-day rehospitalization data were derived from the results of the Heart Failure Registry of Patient Outcomes (HERO) study and the China HF study respectively ${ }^{[21,22]}$. The monthly transition probabilities are presented in Table 1 . These data do not have a distribution in the model, because these were not available. Nevertheless, all-cause death, inhospital death, and rehospitalization are based on large numbers and inherent uncertainty may be small. The hazard ratios(HR) from PARADIGM-HF ${ }^{[8,23,24]}$ were multiplied by the different probabilities (hospital admissions, rehospitalization and deaths) to calculate the parameters for SAC/VAL. The values are presented in Table 2.

\section{Cost}

From the perspective of health care system, only the direct medical cost of interventions is calculated, including the cost of drugs and the cost of hospitalization due to acute heart failure; the cost of drugs is based on the latest national negotiation price of SAC/VAL and enalapril in 2020, which is $¥ 9.95$ per

$100 \mathrm{mg}$ and $¥ 0.56$ per $10 \mathrm{mg}$; the cost of hospitalization for acute aggravation of heart failure comes from the study of Xuan Jianwei et al. ${ }^{[25]}$ in China: the single average cost of hospitalization is $¥ 39063.88 \pm$ 
55343 including the cost of other drugs,diagnsis, operation, examination ,bed and other items(see Table $3)$.

\section{Utilities}

In this study, quality adjusted life year (QALY) is used as the effect index. QALY is calculated by multiplying the health utility value of the patient's state by the time when the patient is in the state. Among them, the health utility value of the healthy patient is assumed to be 1 , and the health utility value of the dead state is 0 ; Due to the lack of literature on the utility value of stable heart failure patients and stable heart failure patients with acute aggravation in China, the previously published EuroQol fivedimensional questionnaire data from Korea are used, the average value of utility scores is calculated from Hong's study. which is closer to the Asian characteristics to measure the relevant utility value data of heart failure in Korea ${ }^{[26]}$ (see Table 4).

\section{Discounting and Willingness to Pay}

This study is a long-term simulation, and the cost and utilities are all based on the discount rate of $5 \%$ recommended by China pharmacoeconomics guide ${ }^{[27]}$. According to the recommendation of the World Health Organization $(\mathrm{WHO})$ on the evaluation of Pharmacoeconomics ${ }^{[28]}$ : ICER $<1$ fold of gross domestic product (GDP) per capita, the increased cost is completely worth it and very cost-effective; 1 fold of GDP per capita < ICER < 3 fold of GDP per capita, the increased cost is acceptable and cost-effective; ICER > 3 fold of GDP per capita, the increased cost is not worth it and not cost-effective. According to the data released by the National Bureau of statistics, Chinese per capita GDP in 2019 is $¥ 708922^{[29]}$ \According to the recommendation of $\mathrm{WHO}$ and China pharmacoeconomics guide, we use three times of per capita GDP ( $¥ 212676$ per QALY) in 2019 as the threshold standard and the willingness to pay (WTP) to judge whether a health intervention is cost-effective.

\section{Sensitivity Analyses}

To investigate the effects of uncertainty in the model, univariate sensitivity analyses were performed by changing the relevant variables and their ranges. The range was taken from $95 \%$ confidence interval from the reported literature ${ }^{[8,21-26]}$. The results of each parameter on ICER were displayed as a tornado diagram. In addition, probability sensitivity analysis(PSA) was also carried out to investigate the uncertainty of all parameters at the same time. This was achieved by calculating the results of 1000 random samples with different parameter distribution, which are transformed into cost-effectiveness acceptability curves (CEACs).

\section{Results}

\section{Model Validation and Clinical Results}


The average age of the simulated population in this study was $63.8 \pm 11.5$ years old. At the end of the PARADIGM-HF trial, with a mean follow-up of 27 months, $17 \%$ of the SAC/VAL and $19.8 \%$ of the enalapril group had died respectively. In our study, at 27 months, we predicted that $18.3 \%$ of the SAC/VAL population and $21.6 \%$ of the enalapril population would die. The average survival time in the model was estimated to be 6.1 years in the enalapril group and 7.5 years in the SAC/VAL group (see figure2). PARADIGM-HF investigators estimated the survival times of a 65 -year-old at 10.2 years for those enalapril group and 11.5 years for those SAC/VAL group ${ }^{[30]}$.

\section{Cost-effectiveness Analysis}

The 10-year treatment cost was $¥ 34560$ of enalapril group and $¥ 96532$ of SAC/VAL group, the total health utility value was 4.3QALY of enalapril group and 4.6QALY of SAC/VAL group, and the ICER was $\Delta$ cost / $\Delta$ utility $=185720$ yuan per QALY, which was less than 3 times of Chinese per capita GDP in 2019.

\section{Sensitivity Analyses}

The results of the univariate sensitivity analysis were shown in the Tornado diagram in Figure 3 . The parameters were ordered with the most influential factor at the top to the least important factor of the results at the bottom. The effect of SAC/VAL on death had the most effect on the ICER which was more than 3 times of Chinese per capita GDP in 2019. The model responded less to changes in any other values, which was less than 3 times of Chinese per capita GDP in 2019.

Different scenario analyses were compared with the base case. Table 6 presented a comparison of the different costs of SAC/VAL at a WTP thresholds of $¥ 212676$ per QALY, which showed that the price of SAC/VAL was not an important factor to affect the cost-effectiveness.

In this study, we assumed that the cost of hospitalization and drugs followed the $y$ distribution, HR of hospitalization, HR of rehospitalization and HR of all-cause death followed the lognormal distribution, the utility value followed the lognormal distribution, PSA of cost, probability and utility value was shown in Figure 4, At the national negotiation price, probability of SAC/VAL being cost-effective was about $53 \%$ at a WTP threshold of $¥ 212676$ per QALY.

\section{Discussion}

From January 1,2020 , the price of SAC/VAL has been reduced from $¥ 17.33$ per 100 mg to $¥ 9.95$ per 100 $\mathrm{mg}$, greatly improving the drug use rate and bringing good news to HF patients in China.

As far as we know, this study is the first time to evaluate the economy of the treatment of HFrEF patients with SAC/VAL after national negotiation price in China. China HF study, CN HF study and HERO study make the epidemiological data of HF patients in China more comprehensive and authoritative ${ }^{[21,22]}$. With the exception of the parameters related to SAC/VAL and the hospitalization rate of enalapril, all data are 
almost from the epidemiologic data of HF patients in China. Our analysis based on Markov model shows that: at the national negotiation price ( $¥ 9.95$ per $100 \mathrm{mg}$ ), SAC/VAL was associated with clinical benefit and may be cost-effective compared with an ACEI (the current standard of care) in patients with HFrEF.

With the exception of the relative risk of dying for SAC/VAL, one-way sensitivity analysis reveals that ICER is all lower than $¥ 212676$ per QALY. The relative risk of dying for SAV/VAL has the most important driver on the cost-effectiveness, which means that avoiding deaths in the SAC/VAL treatment group lead to more QALYs compared with enalapril. The second driver is the health utility value of the stable heart failure and the relative risk of hospital for SAC/VAL because of the additional gained QALYs by avoiding hospital and living longer, the price of SAC/VAL has little impact on the ICER.

The scenario analysis shows that the pre negotiation price of SAC/VAL has a decisive effect on the costeffectiveness and the negotiation price of SAC/VAL has little impact on the cost-effectiveness. The longterm impact of SAC/VAL including reducing death, reducing the number of hospitalizations and prolonging life expectancy is the fundamental factors to promote the use of drugs.

In our model, the cost of drugs and hospitalization, HR of hospitalization, rehospitalization and all-cause death and the utility value have a distribution, but those data including hospitalization, rehospitalization and all-cause death in enalapril do not have a distribution in the model, because these are not available, but all-cause death, in-hospital death, and rehospitalization are based on large numbers and inherent uncertainty may be small. The PSA of our model shows that probability of SAC/VAL being cost-effective was about $53 \%$ at a WTP threshold of $¥ 212676$ per QALY. It may be that most of our data are from the epidemiological data of HF patients in China. Compared with PARADIGM-HF trial, all-cause mortality, inhospital mortality and readmission rate of HF patients in China are relatively high, which is why our model predicts lower survival rate in 27 month and mean survival time. The cost of drugs in our model may be higher than actual cost, because according to PARADIGM-HF, the average dosage of SAC/VAL and enalapril is $375 \pm 71 \mathrm{mg}, 18.9 \pm 3.4 \mathrm{mg}$ respectively, which is almost two times of actual dosage. The reason that we take the dosage from PARADIGM-HF is to use the HR of hospitalization, rehospitalization and all-cause death from PARADIGM-HF in our model and to not reduce the effect of SAC/VAL.

The results are robust and reliable in general in the sensitivity analyses for several reasons; First, HF is a chronic disease with high morbidity and mortality whose median survival time is less than 5 years ${ }^{[31]}$, within a reasonable price range, treatment that reduces death and hospitalization can be cost-effective; Second, relieving the progress of HF may contribute to the relative improvement of the overall quality of life, because the decline of the quality of life is related to the severity of the disease; Third, the high cost of hospitalization is saved, including general ward care and intensive care unit, and the implantation of high consumable heart assist device is reduced.

In our model, the use of SAC/VAL could lead to more than one year of life for each patient and significant cost savings by avoiding hospitalization. Because of the increase in life expectancy of 8-10 million HF patients in $\mathrm{China}^{[3]}$, the benefits of the use of SAC/VAL could be significant. Considering that up to half of 
the HF patients were accompanied by the reduced ejection fraction, a considerable number of patients were likely to receive SAC/VAL treatment. According to our model, it was assumed that 10 million HFrEF patients in China are treated with SAC/VAL. This strategy could reduce patients in hospital by 300 thousand, deaths by 280 thousand, and the medical cost of the hospitalization by $¥ 9$ billion in two years, which could greatly reduce the burden of Chinese medical security system.

There have been many studies abroad to analyze the cost-effectiveness of the treatment of heart failure with SAC/VAL. Solomon et al. considered it to be cost-effective by calculating the ICER of $\$ 45017$ per QALY at the cost of $\$ 375$ per month for SAC/VAL and $\$ 0.96$ per month for enalapril ${ }^{[32]}$; The team in Dutch also considered it cost effective to spend $£ 5.25$ per day for SAC/VAL and the ICER of $£ 19133$ per QALY ${ }^{[15]}$; Germany also announced that it was cost-effective to spend $£ 5.33$ a day for SAC/VAL and the ICER of $£ 26278$ per QALY ${ }^{[3]}$. It can be seen that the treatment of heart failure with SAC/VAL in many countries was cost-effective, which is consistent with our results. Compared with our model, the cost including drugs and hospitalization and the utility value is much higher than that in our model. In their sensitivity analysis, the price of SAC/VAL has a driving effect on whether it is cost-effective, while our model shows that: SAC/VAL on death has a driving effect on whether it is cost-effective.

There are the following limitations in our study: First, we did not consider the cost of the hospitalization for non-HF in our model, but in the PARADIGM-HF trial, the HR of the hospitalization for non-HF was 0.92 (0.85-0.99), and our analysis results could be conservative; Second, because we can't achieve the data of parameters related to SAC/VAL and health utility values of each transfer state of HF patients in China, we use data form foreign trials to simulate, which may lead to some racial bias in the simulation results; Third, we assumed that HF patients in China could tolerate the recommended dose of each drug, regardless of the adverse events, in the PARADIGM-HF trial, the most common adverse events included hypotension, heart failure, hyperkalemia, renal damage and cough, but the proportion of discontinuation of the trial $(10.7 \%)$ due to the occurrence of the adverse events in the SAC/VAL was lower than that of enalapril (12.3\%), which further emphasized our analysis could be conservative;Fourth, the cost of other treatments such as B blocker, aldosterone receptor antagonist, diuretic, digitalis, ICD and cardiac resynchronization were not considered in the calculation of drug cost, but the PARADIGM-HF trial showed no significant difference between the two groups;Fifth, the NYHA classification was not incorporated in the model because these data were not available;Sixth, Productivity losses were not taken into account for this model. because this study was from the health care payer's perspective and not from the societal perspective. Nevertheless, most patients werelikely to be retired when HF is diagnosed, the effect of this is likely to be low.

\section{Conclusion}

In conclusion, the results of pharmacoeconomic evaluation using Markov model in this study show that at the national negotiation price ( $¥ 9.95$ per $100 \mathrm{mg}$ ), SAC/VAL was associated with clinical benefit and may be cost-effective compared with an ACEI (the current standard of care) in patients with HFrEF. 


\section{Abbreviations}

QALY

quality-adjusted life year;

ACEI

angiotensin converting enzyme inhibitor;

HFrEF

heart failure with reduced ejection fraction;

SAC/VAL

sacubitril/valsartan;

ICER

the incremental cost-effectiveness ratio;

PARADIGM-HF

the Prospective Comparison of ARNI (angiotensin receptor-neprilysin inhibitor) with ACEI (angiotensin-

converting enzyme inhibitor) to Determine Impact on Global Mortality and Morbidity in Heart Failure Trial;

WTP

the willingness to pay;

ARB

angiotensin receptor antagonists;

ARNI

angiotensin receptor-neprilysin enzyme inhibitor;

$\mathrm{CN}-\mathrm{HF}$

the China National Heart Failure Registration Study;

HERO

the Heart Failure Registry of Patient Outcomes;

HR

Hazard ratio;

WHO

the World Health Organization;

GDP

Gross Domestic Product;

CEACs

cost-effectiveness acceptability curves;

\section{Declarations}

\section{Ethics approval and consent to participate}

Not applicable.

Consent for publication 
Not applicable.

\section{Competing interests}

The authors declare that they have no competing interests.

\section{Founding}

The authors declare that they have no founding

\section{Authors' contributions}

Zhe WANG and Rujie ZHENG collected the data regarding heart failure; Yaohui JIANG analyzed the data and developed a Markov model; Haiqiang SANG was the leader of the study. All authors have read and approved the final manuscript.

\section{References}

1. Conrad N, Judge A, T ran J, Mohseni H, Hedgecott D, Crespillo AP, Allison M, Hemingway H, Cleland JG, McMurray JJ, Rahimi K. T emporal trends and patterns in heart failure incidence: a populationbased study of 4 million individuals. Lancet. 2018;391:572-80.

2. Gu D, Huang G, He J. etal.Investigation of prevalence and distributing feature of chronic heart failure in China adult popuation. Chin J Cardio. 2003;1(31):3-6.

3. Yang Y, Ma Y, Liu F. etal.Incidence and distributing feature of chronic heart failure in adult population in Xinjiang. Chin J Cardio. 2010;38(5):460-4.

4. The US Centers for Disease Control and Prevention. CDC in China:factsheet. Centers for Disease Controland Prevention. Atlanta, USA.

5. http://. Accessed July 21, 2016.

6. Cook C, Cole G, Asaria P, et al. The annual global economic burden of heart failure. Int J Cardiol. 2014;171:368-76.

7. Mozaffarian D, Benjamin EJ, Go AS, et al American Heart Association Statistics Committee and Stroke Statistics Subcommittee. Heart disease and stroke statistics-2016 update: a report from the American Heart Association. Circ J. 2016;133:e38-60.

8. Desai AS, Stevenson LW. Rehospitalization for heart failure: predict or prevent? Circ J. 2012;126:501-6.

9. McMurray JJ, Packer M, Desai AS, et al. PARADIGM-HF Investigators and Committees. Angiotensinneprilysin inhibition versus enalapril in heart failure. N Engl J Med. 2014;371(11):993-1004.

10. Ponikowski P, Voors AA, Anker SD, et al. 2016 ESC Guidelines for the diagnosis and treatment of acute and chronic heart failure: The Task Force for the diagnosis and treatment of acute and chronic heart failure of the European Society of Cardiology(ESC)Developed with the special contribution of the Heart Failure AssociationロHFAワof the ESC. Eur Heart J. 2016;37(27):2129-200. 
11. Yancy CW, Jessup M, Bozkurt B, et al. 2017 ACC/AHA/HFSA Focused Update of the 2013 ACCF/AHA Guideline for the Management of Heart Failure:A Report of the American College of Cardiology/American Heart Association Task Force on Clinical Practice Guidelines and the Heart Failure Society of America. Circ J. 2017;136(6):e137-61.

12. Group of heart failure, branch of Cardiology, Chinese Medical Association. Professional committee of heart failure, Editorial Committee of Chinese Journal of Cardiology, Guidelines for diagnosis and treatment of heart failure in China. Chin J Cardio. 2018;46(10):760-89.

13. Sandhu AT, Ollendorf DA, Chapman RH, et al. Cost-effectiveness of sacubitril-valsartan in patients with heart failure with reduced ejection fraction. Ann Intern Med. 2016;165(10):681-9.

14. King JB, Shah RU, Bress AP, et al. Cost-effectiveness of sacubitril-valsartan combination therapy compared with enalapril for the treatment of heart failure with reduced ejection fraction. JACC: Heart Failure. 2016;4(5):392-402.

15. Gaziano TA, Fonarow GC, Claggett B, et al. Cost-effectiveness analysis of sacubitril/valsartan vs enalapril in patients with heart failure and reduced ejection fraction. JAMA cardiology. 2016;1(6):666-72.

16. van der Pol S, Degener F, Postma MJ, et al. An economic evaluation of sacubitril/valsartan for heart failure patients in the Netherlands. Value in Health. 2017;20(3):388-96.

17. Ramos IC, Versteegh MM, de Boer RA, et al. Cost effectiveness of the angiotensin receptor neprilysin inhibitor sacubitril/valsartan for patients with chronic heart failure and reduced ejection fraction in the Netherlands: a country adaptation analysis under the former and current Dutch Pharmacoeconomic Guidelines. Value in Health. 2017;20(10):1260-9.

18. Ademi Z, Pfeil AM, Hancock E, et al Cost-effectiveness of sacubitril/valsartan in chronic heart-failure patients with reduced ejection fraction. Swiss medical weekly, 2017: 147: w14533. [18] Liang BinChia L, Wu D, Aziz MIA, et al. Cost-effectiveness of sacubitril/valsartan versus enalapril in patients with heart failure and reduced ejection fraction. Journal of medical economics, 2018, 21(2): 174181.

19. Zhou J, Cao J, Jin X, et al. Digoxin is associated with worse outcomes in patients with heart failure with reduced ejection fraction. ESC Heart Fail. 2020. .

20. Digitizeld. Digitizelt-graph digitizer software. Digitize graphs, charts and math data. Available from: .

21. Li L, Liu R, Jiang C, et al. Assessing the evidence-practice gap for heart failure in China: the Heart Failure Registry of Patient Outcomes (HERO) study design and baseline characteristics. Eur J Heart Fail. 2019. .

22. Zhang Y, Zhang J, Butler J, et al. Contemporary epidemiology, management, and outcomes of patients hospitalized for heart failure in China: results from the China heart failure (China-HF) registry[J]. J Card Fail. 2017;23(12):868-75.

23. Desai AS, Claggett BL, Packer M, et al. Influence of sacubitril/valsartan (LCZ696) on 30-day readmission after heart failure hospitalization. J Am Coll Cardiol. 2016;68(3):241-8. 
24. Packer M, Mcmurray JJ, Desai AS, et al. Angiotensin receptor neprilysin inhibition compared with enalapril on the risk of clinical progression in surviving patients with heart failure. Circ J. 2015;131(1):54-61.

25. Xuan J, Tao L, Zhu S, et al. Real World Retrospective Chart Review Study of the Hospitalization Costs and Associated Risk Factors for Heart Failure Patients in China. China Health Insurance(Chinese), 2017(3):61-64.

26. Hong SH, Lee JY, Park SK, et al. The utility of 5 hypothetical health states in heart failure using time trade-off (TTO) and EQ-5D-5L in Korea. Clinical drug investigation. 2018;38(8):727-36.

27. Research group of China Pharmacoeconomics Evaluation. China Guidelines for Pharmacoeconomic Evaluations. (2011). China Pharmacoeconomics(Chinese),20117(3):6-9,1148.

28. World Health Organization. Making choices in health: WHO guide to cost-effectiveness analysis. Geneva: World Health Organization. 2003;9. .

29. National Bureau of statistics of the people's Republic of China

China Statistical Yearbook, 2019[DB/OL]

$01 \& z b=A 0201 \& s j=2019$

National Bureau of statistics of the people's Republic of China. China Statistical Yearbook, 2019[DB/OL]. [2019-3-27]. 01\&zb=A0201\&sj=2019.

30. Claggett B, Packer M, Mcmurray J, et al. PARADIGM-HF Investigators. Estimating the long-term treatment benefits of sacubitril-valsartan. N Engl J Med. 2015;373(23):2289-90.

31. Stewart S, Macintyre K, Hole, Dj, et al. More 'malignant' than cancer? Five-year survival following a first admission for heart failure. Eur J Heart Fail. 2001;3(3):315-22.

32. Gaziano TA, Fonarow GC, Claggett B, et al. Cost-effectiveness Analysis of Sacubitril/Valsartan vs Enalapril in Patients With Heart Failure and Reduced Ejection Fraction. JAMA cardiology. 2016;1(6):666-72.

33. Van Der Pol S, Degener F, Postma MJ, et al. An Economic Evaluation of Sacubitril/Valsartan for Heart Failure Patients in the Netherlands. Value in health: the journal of the International Society for Pharmacoeconomics Outcomes Research. 2017;20(3):388-96.

34. Akshay SDesai, John JVMcmurray, Packer M, et al. Effect of the angiotensin-receptor-neprilysin inhibitor LCZ696 compared with enalapril on mode of death in heart failure patients. Eur Heart J. 2015;36:1990-7.

\section{Tables}

Table 1 - Transition probabilities used in the Markov model.

\begin{tabular}{ccc}
\hline Transition & Probability & Source \\
\hline Hospitalization & 0.0121 & {$[8]$} \\
Death $\llbracket$ all cause $\square$ & 0.0089 & {$[19]$} \\
Death(in hospital) & 0.041 & {$[22]$} \\
Rehospitalization $\llbracket$ enalapril $\square$ & 0.155 & {$[21]$} \\
\hline
\end{tabular}


Table 2 Effects (Hazard ratio) of sacubitril/valsartan compared with enalapril.

\begin{tabular}{ccc}
\hline Target of effect & Risk ratio (sacubitril/valsartan compared with enalapril) $(95 \%$ CI) & Source \\
\hline Hospitalization & $0.77(0.67-0.89)$ & {$[8,24]$} \\
Death & $0.84(0.76-0.93)$ & {$[8]$} \\
Rehospitalization & $0.62(0.45-0.87)$ & {$[23]$} \\
\hline
\end{tabular}

Table 3 Medicine costs for the drugs and usage in the model.

\begin{tabular}{lcc}
\hline \multicolumn{1}{c}{ Projects } & Cost per month(¥) & Source \\
\hline Enalapril & 1139.38 & national negotiation price \\
SAC/VAL & 31.54 & national negotiation price \\
Hospitalization & 39063.88 & {$[25]$} \\
\hline
\end{tabular}

Table 4 Utilities of event used in the Markov model

\begin{tabular}{ccc}
\hline Projects & Utilities & Source \\
\hline Hospitalization & $0.215 \pm 0.173$ & {$[26]$} \\
Stable heart failure & $0.871 \pm 0.088$ & {$[26]$} \\
Death & 0 & {$[26]$} \\
\hline
\end{tabular}

Table 5 Results of one-way sensitivity analyses, The range was taken from 95\% confidence interval .

\begin{tabular}{ccccc}
\hline Parameter & \multicolumn{2}{c}{ 95\%CI } & \multicolumn{2}{c}{ ICER(¥ per QALY) } \\
\cline { 2 - 5 } & Low value & High value & Low value & High value \\
\hline SAC/VAL on death & 0.760 & 0.930 & 132207.990 & 358927.070 \\
Utility:nohospital & 0.717 & 1.00 & 226098.270 & 161553.650 \\
SAC/VAL on hospital & 0.670 & 0.890 & 170522.650 & 205339.250 \\
SAC/VAL on rehospital & 0.450 & 0.870 & 182355.070 & 191137.740 \\
Cost:hospital & 33370.560 & 44757.198 & 188911.140 & 182530.610 \\
Utility:hospital & -0.066 & 0.676 & 183315.490 & 189806.830 \\
Cost:enalapril & 31.370 & 31.710 & 185751.330 & 185690.650 \\
Cost:SAC/VAL & 1112.950 & 1125.800 & 184497.980 & 185751.330 \\
Discount & 0.021 & 0.083 & 185720.879 & 185720.879 \\
\hline
\end{tabular}

Table 6 -ICER from costs of SAC/VAL for different scenarios.

\begin{tabular}{ccc}
\hline Different scenarios & Cost per month(¥) & ICER(¥ per QALY) \\
\hline negotiation price & 1119.375 & 185720.879 \\
Pre negotiation price & 1949.223 & 343760.396 \\
Price reduction $20 \%$ & 895.500 & 143085.254 \\
Price reduction $40 \%$ & 671.625 & 100449.630 \\
Price reduction $60 \%$ & 447.750 & 57814.005 \\
\hline
\end{tabular}




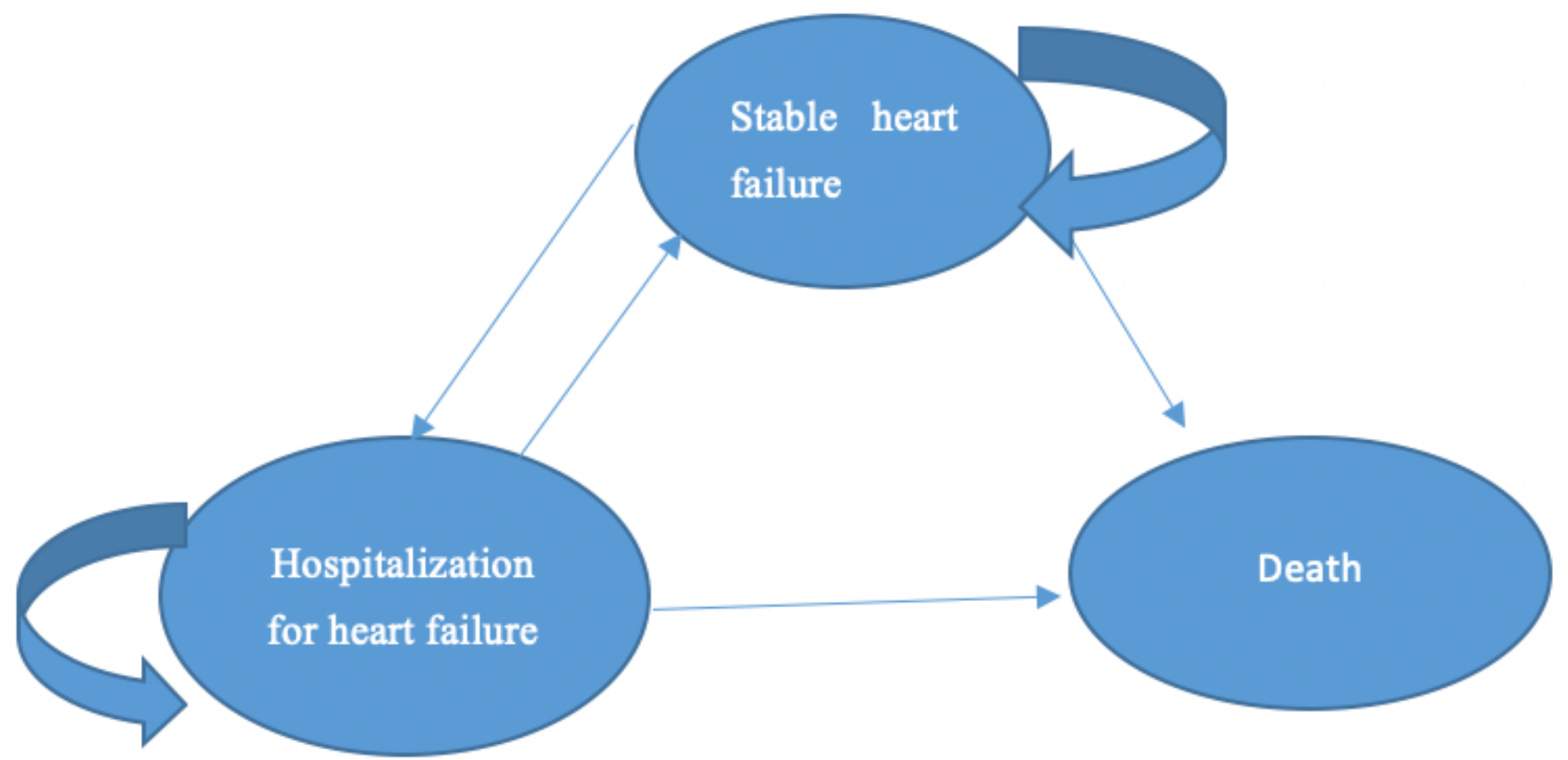

\section{Figure 1}

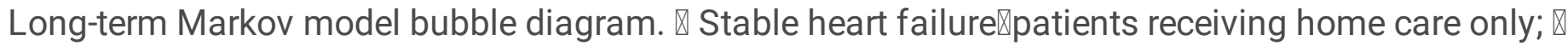
Hospitalization: hospitalization for acute aggravation of heart failure; $₫$ Death: all cause death. 


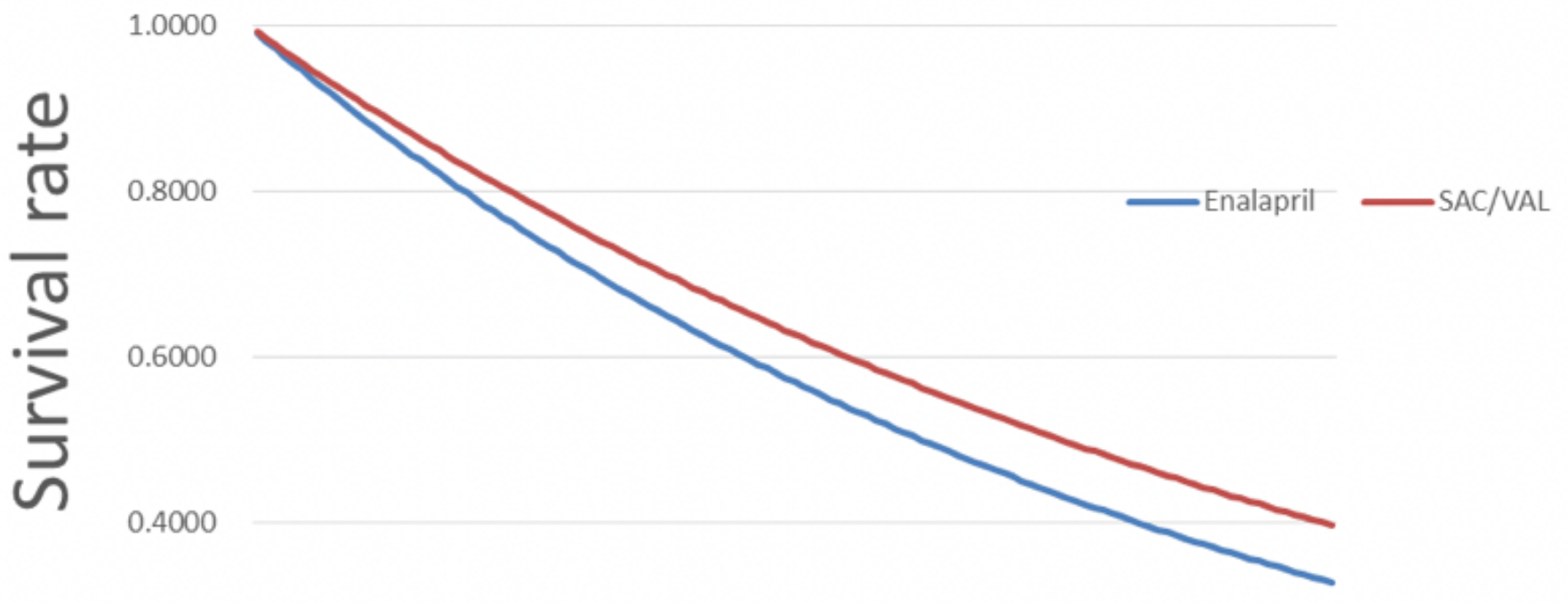

0.2000

0.0000 Months

Figure 4

Survival Curves, According to Treatment Strategy, Shown are estimates of the probability of being alive at any given time over 120 months. 
$¥ 0.00 \quad ¥ 500.00 ¥ 1,000.00 ¥ 1,500.00 ¥ 2,000.00 ¥ 2,500.00 ¥ 3,000.00 ¥ 3,500.00 ¥ 4,000.00$

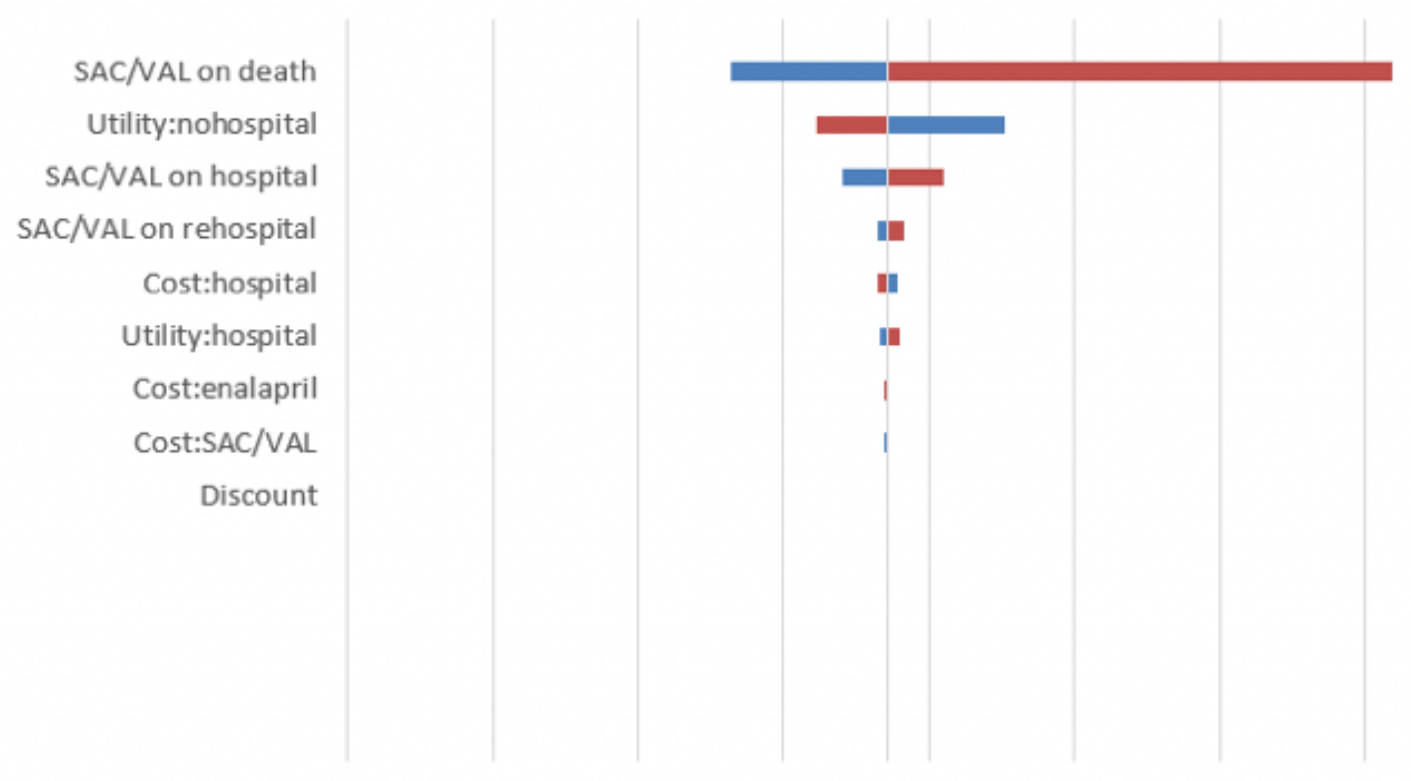

- Low value High value

\section{Figure 6}

Tornado diagram showing the univariate sensitivity analysis of the Markov model simulation. The range was taken from $95 \%$ confidence interval . 


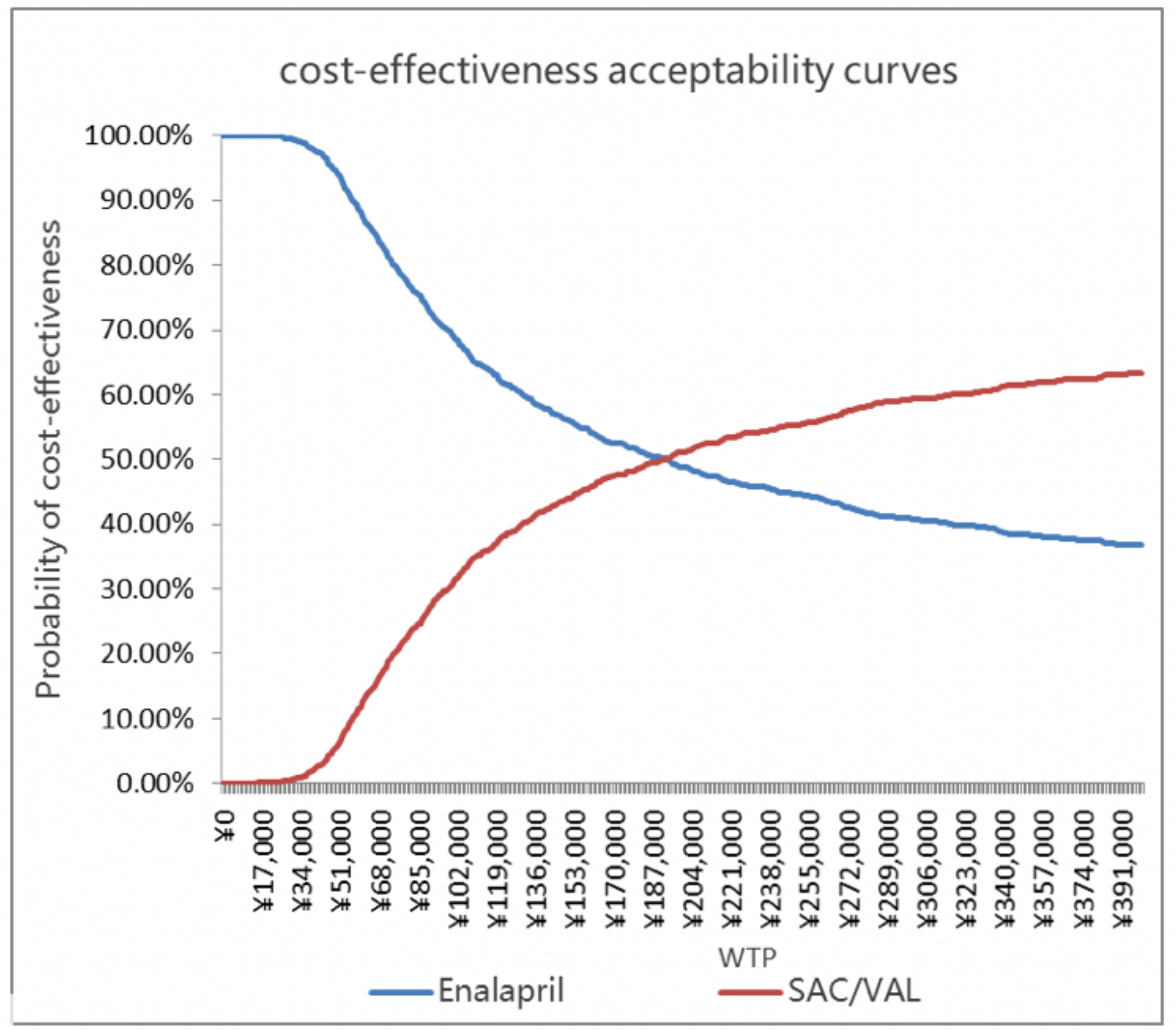

Figure 8

Cost-effectiveness acceptability curve showing the maximum willingness to pay (or threshold ratio) and the corresponding probability of cost-effectiveness for both enalapril and SAC/VAL. 